\title{
MICROMECHANICS OF SINGLE SUPERCOILED DNA MOLECULES
}

\author{
JOHN F. MARKO*
}

\begin{abstract}
The theory of the mechanical response of single DNA molecules under stretching and twisting stresses is reviewed. Using established results for the the semiflexible polymer including the effect of torsional stress, and for the free energy of plectonemic supercoils, a theory of coexisting plectonemic and extended DNA is constructed and shown to produce phenomena observed experimentally. Analytical results for DNA extension and torque are presented, and effects of anharmonicities in the plectonemic free energy are described. An application of the theory to the problem of torsional-stress-induced cruciform extrusion is also discussed.
\end{abstract}

Key words. DNA, molecular biology, statistical mechanics, polymer physics.

AMS(MOS) subject classifications. 82D60, 92C05, 92C40.

1. Introduction. Single-molecule stretching is a powerful tool not just for the study of the DNA double helix, but also for the study of proteins which interact with it. A wide variety of such experiments have followed from seminal DNA-stretching work of Smith et al. [1]. The semiflexible polymer model provides a quantitative starting point for theories describing these types of experiments $[2-5]$. This model, which describes DNA bending fluctuations in terms of a single "persistence length", is useful thanks to the separation of the double-helix persistence length $(50 \mathrm{~nm})$ from both the base-pair scale $(0.34 \mathrm{~nm})$ and the total molecular length scale ( $>1000 \mathrm{~nm}$ for $>3 \mathrm{~kb}$ molecules; $1 \mathrm{~nm}=10^{-9} \mathrm{~m}$ ). The availability of a simple but quantitative theoretical framework for DNA elasticity has greatly facilitated analysis of a wide variety of single-DNA-based experiments.

DNA's double-helix structure gives it a twist modulus. Both this twisting elasticity, and the topology of wrapping of the DNA strands in the double helix are of paramount biological importance, with whole families of enzymes known to play various roles in control of and response to DNA linking number in vivo. Many of these enzymes, including site-specific recombinases, topoisomerases, nucleic acid polymerases, and the mysterious "structural maintenance of chromosome" cohesin and condensin protein complexes, had their functions dissected by Nick Cozzarelli, to whom this volume is dedicated.

Elegant single-DNA manipulation techniques have been developed to control the linking numbers of individual DNA molecules, and quantitative studies have been made of the elasticity of twisted DNA molecules [6-9]. A dominant feature of the elastic behavior of twisted DNA is that

* Department of Physics and Astronomy and Department of Biochemistry, Molecular Biology and Cell Biology, Northwestern University, Evanston, Illinois 60208-3500. 


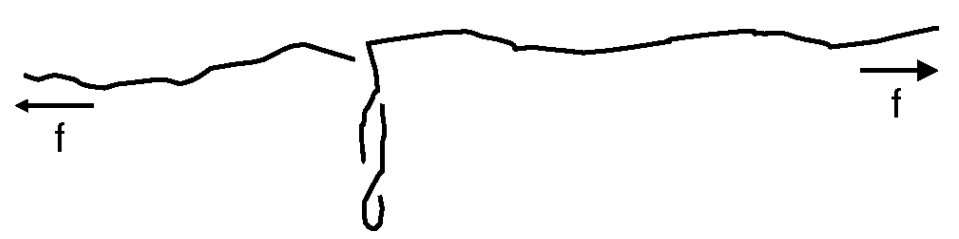

FIG. 1. Sketch of a DNA molecule under tension $f$, and with linking number fixed so as to put the double helix under torsional stress. Over a range of applied tension, the molecule breaks up into "domains" of extended and plectonemically supercoiled DNA.

at fixed force, introduction of sufficient linking number to a DNA causes it to start to wrap around itself or supercoil in the manner of a twisted wire. This buckling is driven by the reduction of twist elastic energy occurring when linking number is transferred to chiral bending, or "writhe". Further introduction of linking number causes folding up of the molecule into a plectonemic supercoiled DNA, and a gradual reduction in molecule extension. This folding can be discussed in terms of coexisting domains of extended and supercoiled DNA [10-16], as sketched in Fig. 1.

An important feature of this domain, or phase-coexistence-like behavior is that as linking number is converting DNA between plectonemic and extended form at a constant force, the torque in the molecule is constant $[8,9]$. In such experiments, the torques applied to single DNAs are typically on the order of a few $k_{B} T$ or $\mathrm{pN} \cdot \mathrm{nm}\left(1 \mathrm{pN}=1\right.$ piconewton $=10^{-12}$ newton; $k_{B} T=4.1 \mathrm{pN} \cdot \mathrm{nm}$ at $\left.T=300 \mathrm{~K}\right)$.

Precise measurements of elasticity of twisted DNAs have been used as the basis of studies of enzymes whose interactions with DNA depend on that twisting, notably topoisomerases [17-23]. Nick Cozzarelli played a key role in many of these experiments, as well as in single-molecule experiments examining basic twisting elasticity of DNA [24, 25].

This paper presents a brief review of the theoretical description of elastic properties of twisted DNA molecules. Of particular interest is the question of exactly what the torque is in a twisted stretched DNA and how it is related to other measurable properties of the molecule, since torque drives linking number relaxation in the experiments mentioned above. New optical tweezer techniques using rotationally polarized light promise to bring direct and precise torque measurements [26].

Sec. 2 reviews results of the statistical mechanical semiflexible polymer theory used to describe DNA stretching. Then, Sec. 3 discusses how the theory can be modified to describe twisted DNA molecules, using a mixedstate treatment of plectonemic and extended-twisted molecule domains. Sec. 3.4 reviews recently published results for that theory [27], while Sec. 4 presents an extension to that model which should permit precise description of experimental data. Sec. 5 discusses an application of the same general 
type of theory to the problem of extrusion of a cruciform structure from DNA containing a palindromic sequence. Finally the Conclusion discusses prospects for further application and development of the theory.

2. The DNA double helix is a semiflexible polymer with twist rigidity. In this section the basic polymer physics of the DNA double helix are reviewed (for a pedagogical introduction, see Ref. [28]). Thanks to its not-too-large bending stiffness, few-kilobase-long DNAs have conformational fluctuations that are well described by a single bending stiffness, or "bending persistence length". The double helical structure of the molecule gives the double helix the additional feature of a twist rigidity.

2.1. Persistence length and polymer stretching elasticity. Molecules of double-helix DNA under physiological solution conditions (aqueous solution with $\mathrm{pH}$ buffered to be near 7.5, salt concentration in the $10 \mathrm{mM}$ to $500 \mathrm{mM}$ range, temperature between 20 and $37^{\circ} \mathrm{C}$ ) behave as semiflexible polymers, with a well-defined bending persistence length of $A \approx 50 \mathrm{~nm}$ [29]. Recalling that there are just about 3 base pairs (bp) per $\mathrm{nm}$ of contour length, this indicates that an otherwise unconstrained DNA molecule in solution undergoes thermally excited bending causing its local tangent to reorient every $\approx 150 \mathrm{bp}$. However, if tension is applied to the molecule, the tangent vector will align with that applied tension. Here, molecules which are many persistence lengths long are of interest, i.e. in the multi-kilobase range, the typical size of circular plasmid molecules or chromosome loop domains in bacteria.

For forces well below $k_{B} T / A$ a DNA molecule will be only slightly stretched; for forces well in excess of $k_{B} T / A$ the molecule stretches out. Given that $k_{B} T \approx 4.1 \mathrm{pN} \cdot \mathrm{nm}$, this threshold force for stretching a doublehelix DNA is $k_{B} T / A=0.08 \mathrm{pN}$. This is a rather low force by molecular biological standards: most molecular motors use chemical energy of a few $k_{B} T$ to take steps of a few $\mathrm{nm}$ in length, so that the scale for molecular motor forces is on the scale of a few $\mathrm{pN}$. The low value of $k_{B} T / A$ is due to the long persistence length of the double helix $(A=50 \mathrm{~nm})$. In contrast single-stranded nucleic acid molecules have a much shorter persistence length $(\approx 1 \mathrm{~nm})$ and consequently do require few-pN forces for their full extension [1]. Thus, double-helix DNAs can be easily stretched out to their full extension by molecular motors in the cell; single-stranded DNA (or RNA, or denatured proteins) require larger forces to be stretched out.

The semiflexible polymer model has been shown to give a good account of the stretching properties of multi-kilobase DNAs from forces of below 0.1 $\mathrm{pN}$ up to roughly $20 \mathrm{pN}[2,3,5]$. For higher forces the secondary structure of the double helix starts to be deformed $[4,5]$, eventually leading to an abrupt first-order-like "overstretching" transition whereby the double helix contour length increases by about $70 \%[30,31]$.

The statistical mechanics of the semiflexible polymer model can be solved to arbitrary accuracy numerically; the asymptotic high- and low- 
force limits are understood analytically [5]. The global variation of the force $f$ as a function of extension $X$ is captured well by the expression

$$
f=\frac{k_{B} T}{A}\left[\frac{X}{L}+\frac{1}{4(1-X / L)^{2}}-\frac{1}{4}\right]
$$

where $L$ is the total molecule contour length (recall that there are $0.34 \mathrm{~nm}$ of contour length per base pair, so that a $10 \mathrm{~kb}$ molecule is about 3000 $\mathrm{nm}=3 \mu \mathrm{m}$ in contour length). Eq. 2.1 has the correct low-extension $(X / L<<1)$ linear elasticity response. For high extensions $(X / L \rightarrow 1)$ the force increases drastically due to an increasing entropic cost of quenching thermal bending fluctuations with progressively smaller wavelengths. An even more accurate approximate representation of the exact solution is obtained by adding a term $-(3 / 4)(X / L)^{2}$ inside the square brackets [32], eliminating any low-extension $X^{2}$ dependence which should be absent via symmetry considerations [11].

The integral of force over extension, or $W(X)=\int_{0}^{X} d X^{\prime} f\left(X^{\prime}\right)$, gives the work done stretching a DNA at constant temperature or the free energy for the semiflexible polymer as a function of extension [11]. In what follows the free energy at fixed force is needed, which is obtained via the Legendre transformation $g(f)=f X-W(X)$; the extension $X$ is eliminated using the inverse of (2.1) [11]. The leading behavior of $g(f)$ and the average extension $X=\partial g / \partial f$ behaves asymptotically for high force as

$$
\begin{aligned}
& g(f)=f-\sqrt{k_{B} T f / A}+\cdots \\
& X(f)=1-\sqrt{\frac{k_{B} T}{4 A f}}+\cdots
\end{aligned}
$$

For the DNA double helix, these leading terms are sufficient for situations where forces are between $0.2 \mathrm{pN}$ and $10 \mathrm{pN}$, which applies over much of the force range for single-DNA stretching experiments. To describe slightly higher forces up to $40 \mathrm{pN}$, linear contour length stretching elasticity may be added $[4,11]$.

2.2. Twisting stiffness of the double helix. If one could twist a DNA molecule without bending it, one could measure the work required to introduce a total twist by an angle $\Theta$, or the twisting free energy. In the absence of bending, $\Theta$ would correspond to the change in linking number of the double helix (number of wraps of one strand around the other), via $\Delta \mathrm{Lk}=\Theta / 2 \pi$. Molecular biologists often describe linking number changes normalized by the linking number of the relaxed double helix, $\mathrm{Lk}_{0}=L / h$ where the helix repeat is $h=3.6 \mathrm{~nm}=10.5 \mathrm{bp}: \sigma=\Delta \mathrm{Lk} / \mathrm{Lk}_{0}$. In the bacterium $E$. coli, it is known that circular plasmid and chromosomal DNAs have $\sigma \approx-0.05$, i.e., their DNAs have about $5 \%$ fewer links than would be expected from their contour length. 
For small pure twists, the energy per contour length is expected by symmetry [10] to be that of a twisted elastic rod [33]:

$$
\frac{E_{\mathrm{twist}}(\sigma)}{L}=\frac{k_{B} T C}{2}\left(\frac{2 \pi \Delta \mathrm{Lk}}{L}\right)^{2}=\frac{k_{B} T C \omega_{0}^{2}}{2} \sigma^{2}
$$

where $C$ is the twist persistence length of DNA (so that $k_{B} T C$ is the twist rigidity elastic constant [33]), and where $\omega_{0}=2 \pi / h=1.85 \mathrm{~nm}^{-1}$ is the spatial rate of circulation of the relaxed double helix.

This form of simple twist energy has been shown to apply to DNA for small linking number changes in direct micromechanical measurements as well as in biochemical studies. However, precise measurement of the twist rigidity $C$ of the double helix has proven to be problematic, with different types of experiments yielding different results [34]. Even among singlemolecule mechanical experiments there have been disagreements between different analyses of experimental data [35].

A cause of difficulty in determining $C$ precisely has been that experiments where DNA is twisted are always subject to chiral bending effects. First, even for small amounts of twisting, chiral bending fluctuations cause a shift (or "renormalization") of the apparent twist rigidity; the energetic cost of twisting the double helix can be reduced by chiral bending fluctuations [36, 37]. A second complication is that for larger amounts of twisting, plectonemic supercoiling of DNA generates a large amount of writhe which further reduces the twist energy cost of adding linking number [11].

A third problem is that DNA tends to be more easily denatured by untwisting than by overtwisting due to its right-handed helix chirality, and also that twisting is directly coupled to changes in DNA helix contour length [38-41]. This effect, which becomes more important as force is increased, further complicates comparison of experiments done with opposite signs of $\sigma$, as well as fits to microscopic elastic theories which often do not account for chiral asymmetry and denaturation effects. Despite all these challenges, there is a rough consensus between single-DNA experiments in the few-pN ranges that the "bare" twist rigidity of DNA at forces below a few $\mathrm{pN}$ is in the range $C=95 \pm 20 \mathrm{~nm}[36,37,24,35,16]$.

3. Supercoiling and torque in stretched twisted DNA. A general framework is now described, aimed at describing micromechanical experiments on single DNAs, taking into account effects of "coexistence" of domains of DNA in different conformations. The main application discussed in this section is to coexistence of plectonemic supercoils and extended DNA. The calculations here are similar to those discussed in more detail in a recent paper [27]. Here, new results will be presented for the effect of anharmonicity in the plectonemic supercoiling free energy on DNA extension obtained as a function of force and linking number.

3.1. Coexistence of DNA states. If a DNA molecule is stretched by force $f$, its free energy and structure change as its linking number density 
$\sigma$ is changed. Here, the DNA molecule is treated as being divided into domains of two "pure" states, each of which is described by a free energy per molecular length, dependent on applied force $f$ and the linking number density $\sigma$. As the forces on the two domains are equal, the focus will be on the $\sigma$ dependences of the two states, which in most of this paper will describe "stretched" and "plectonemic" DNA [10, 11, 14, 13, 15, 16].

The free energies per length of the pure states are taken to be $\mathcal{S}(\sigma)$ for stretched and $\mathcal{P}(\sigma)$ for plectonemic DNA. From a statistical-mechanical perspective, these free energies are $-k_{B} T$ times the logarithm of the partition function for the molecule at fixed force and linking number, divided by the relaxed double helix contour length; note that all the free energies per length can be converted to free energies per base pair by multiplying by $0.34 \mathrm{~nm} / \mathrm{bp}$. For these pure states, the rate that work is done injecting linking number is proportional to torque:

$$
\tau=\frac{1}{\omega_{0}} \frac{\partial \mathcal{S}(\sigma)}{\partial \sigma} .
$$

The prefactor converts the $\sigma$ derivative to one with respect to rotation angle; a similar equation holds for the $\mathcal{P}$ state.

Along a molecule which is a fraction $x_{\mathrm{s}}$ of state $\mathcal{S}$ and fraction $x_{\mathrm{p}}=$ $1-x_{\mathrm{s}}$ of state $\mathcal{P}$, the free energy per base pair of the mixed phase is

$$
\mathcal{F}(\sigma)=x_{\mathrm{s}} \mathcal{S}\left(\sigma_{\mathrm{s}}\right)+x_{\mathrm{p}} \mathcal{P}\left(\sigma_{\mathrm{p}}\right) .
$$

The equilibrium length fraction $x_{\mathrm{s}}$ and the free energy is determined by minimization of this free energy subject to the constraint $\sigma=x \sigma_{\mathrm{s}}+x_{\mathrm{p}} \sigma_{\mathrm{p}}$.

This last equation indicates that linking number is being partitioned between the two states. This must be justified, since while twist is locally defined, in general writhe cannot be considered as a local variable that can be partitioned in this way. At the workshop, Craig Benham emphasized this point.

However, in the case of interest here, the plectonemic regions are essentially closed loops (see Fig. 1). By "pinching" of those loops off to form circular plectonemic supercoils separated from the extended DNA, the calculation of writhe can be decoupled into separate writhes for extended and plectonemic regions. The "cross terms" ignored by this decoupling correspond to the Gauss linking invariant of the plectonemic regions with one another and with the extended DNA, all of which are zero. The entire chain is an unknot, guaranteeing that this pinching-off decoupling can always be done, but this also indicates that the physically relevant case of unknotted and self-avoiding DNA must be considered. For a "phantom DNA" model lacking the unknot topology constraint, nontrivial knotting of the chain could cause this partitioning to break down [12]. At the workshop de Witt Sumners described a sketch of a proof of the additivity of linking numbers of extended and plectonemic regions in this experimentally relevant unknot case. 


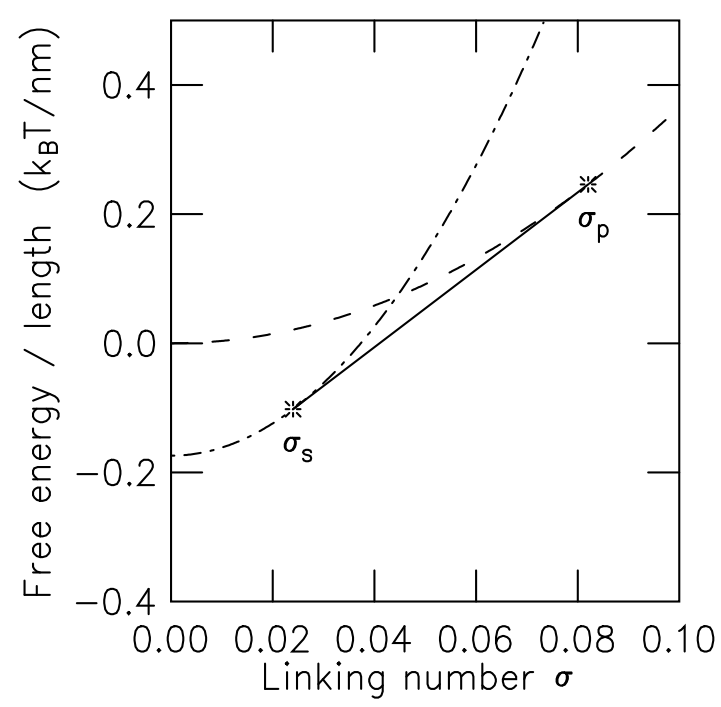

FIG. 2. Free energies of extended (dot-dashed curve, $\mathcal{S}(\sigma)$ ) and plectonemic supercoil (dashed curve, $\mathcal{P}(\sigma)$ ) DNA states as a function of linking number $\sigma$. For $\sigma<\sigma_{\mathrm{s}}$, the $\mathcal{S}$ state is lower in free energy than either $\mathcal{P}$ or any mixture of the two. Similarly, for $\sigma>\sigma_{\mathrm{p}}$, pure $\mathcal{P}$ is the lowest-free energy configuration. However, for $\sigma$ between $\sigma_{\mathrm{s}}$ and $\sigma_{\mathrm{p}}$ the tangent construction shown (solid line segment between tangent points indicated by stars), representing coexisting domains of $\mathcal{S}\left(\sigma_{\mathrm{s}}\right)$ and $\mathcal{P}\left(\sigma_{\mathrm{p}}\right)$, is the lowest free energy state. Note that the gap between the two states at $\sigma=0$ is the free energy difference between random coil DNA $[\mathcal{S}(0)]$ and stretched unsupercoiled DNA $[\mathcal{P}(0)]$; this difference grows with applied force.

If the two pure state free energy densities plotted as a function of linking number density never cross, then one pure state or the other will be the equilibrium state, i.e., one of the two extreme cases $x_{\mathrm{s}}=0$ or $x_{\mathrm{s}}=1$ will always minimize Eq. 3.2. However, if the two free energy densities cross, then there will be a range of $\sigma$ over which there will be coexisting domains of the two states. Fig. 2 shows this situation, sketched to correspond to the case of main interest here, where at low values of $\sigma$ the stretched state is stable (lower in free energy) relative to the plectoneme state, but where at large $\sigma$ the stability reverses due to "screening" of the twist energy by the plectonemic state's writhe [10, 11, 14, 15].

Minimization of Eq. 3.2 is accomplished by a double-tangent construction familiar from other examples of phase coexistence (e.g., liquidgas); in this case the conserved density is that of linking number (Fig. 2). The two coexisting states of linking number densities $\sigma_{\mathrm{s}}$ and $\sigma_{\mathrm{p}}$ satisfy $\partial \mathcal{S}\left(\sigma_{\mathrm{s}}\right) / \partial \sigma_{\mathrm{s}}=\partial \mathcal{P}\left(\sigma_{\mathrm{s}}\right) / \partial \sigma_{\mathrm{p}}$, i.e., they have equal torques. They mix in proportions $x_{\mathrm{s}}$ and $x_{\mathrm{p}}$, so the free energy in the coexistence region is 


$$
\mathcal{F}(\sigma)=\mathcal{S}(\sigma)+\frac{\partial \mathcal{S}\left(\sigma_{\mathrm{s}}\right)}{\partial \sigma_{\mathrm{s}}}\left(\sigma-\sigma_{\mathrm{s}}\right)=\mathcal{P}(\sigma)+\frac{\partial \mathcal{P}\left(\sigma_{\mathrm{p}}\right)}{\partial \sigma_{\mathrm{p}}}\left(\sigma-\sigma_{\mathrm{p}}\right)
$$

In the coexistence region, the fractions of the two states in the mixed state depend linearly on $\sigma$, as

$$
x_{\mathrm{s}}=\frac{\sigma_{\mathrm{p}}-\sigma}{\sigma_{\mathrm{p}}-\sigma_{\mathrm{s}}} \quad x_{\mathrm{p}}=\frac{\sigma-\sigma_{\mathrm{s}}}{\sigma_{\mathrm{p}}-\sigma_{\mathrm{s}}} .
$$

The coexistence construction guarantees that the free energy is a convex function of linking number, and therefore that the torque is a monotonic function of linking number, as required for mechanical stability. In the coexistence region ( $\sigma$ between the limits $\sigma_{\mathrm{s}}$ and $\sigma_{\mathrm{p}}$ ) the torques in the two types of domains are equal and $\sigma$-independent; i.e., the $\sigma$-derivative of Eq. 3.3 is constant.

In the coexistence region Eq. 3.4 indicates that the rate of change of the length fractions with $\sigma$ is constant; $\partial x_{\mathrm{s}} / \partial \sigma=-1 /\left(\sigma_{\mathrm{p}}-\sigma_{\mathrm{s}}\right)$. This generates the linear dependence of molecule extension on linking number observed experimentally once the threshold for generating plectonemic DNA is reached, as can be seen by computing the molecule extension (as a fraction of relaxed double helix contour length $L$ ):

$$
\frac{z}{L}=-\frac{\partial \mathcal{F}}{\partial f}=-x_{\mathrm{s}} \frac{\partial \mathcal{S}\left(\sigma_{\mathrm{s}}\right)}{\partial f}-x_{\mathrm{p}} \frac{\partial \mathcal{P}\left(\sigma_{\mathrm{p}}\right)}{\partial f} .
$$

In the coexistence region, the only $\sigma$ dependence is the linear variation of $x_{\mathrm{s}}$ and $x_{\mathrm{p}}$, making the dependence of extension on $\sigma$ entirely linear.

In the main case of interest here where $\mathcal{P}$ is the plectonemic supercoil state, its zero length eliminates its contribution to Eq. 3.5 ( i.e., $\partial \mathcal{P} / \partial f=$ $0)$, yielding

$$
\frac{z}{L}=-x_{\mathrm{s}} \frac{\partial \mathcal{S}\left(\sigma_{\mathrm{s}}\right)}{\partial f}=\frac{\sigma_{\mathrm{p}}-\sigma}{\sigma_{\mathrm{p}}-\sigma_{\mathrm{s}}} \frac{z\left(\sigma_{\mathrm{s}}\right)}{L}
$$

where the final extension per length factor is the extension per length of the extended DNA state.

Experimentally, $\sigma_{s}$ and $\sigma_{p}$ may be measured from the beginning and the end of the linear coexistence regime of extension as a function of $\sigma$. Likewise, $z\left(\sigma_{\mathrm{s}}\right) / L$ is the extension per length of the molecule at the onset of the linear regime. Thus Eq. 3.6 can be used to determine the coexisting state linking number values, the extension of the stretched DNA state as a function of force and linking number, and via integration the free energy of the stretched state. Then through use of the tangent construction (Fig. 2), the free energy of the plectonemic state can be measured. Note that when the molecule is entirely converted to plectoneme $\left(x_{\mathrm{s}}=0, x_{\mathrm{p}}=0\right)$ the extension reaches zero. The point $\sigma=\sigma_{\mathrm{p}}$ where this occurs can be estimated experimentally from extrapolation of extension data to zero. 
3.2. Free energy of extended twisted DNA. The previous subsection outlines the basic scheme of calculation of equilibrium domain coexistence along a stretched and supercoiled DNA, but to calculate a experimentally relevant result, explicit forms for the extended and plectonemic state free energies are needed. Fortunately, suitable formulae based on statistical-mechanical treatments of microscopic models are available.

For a DNA under torsional stress there occur chiral fluctuations even when the chain is fully extended $[10,11,36,37]$. The free energy of the extended DNA at a fixed force $f$ can be written as an expansion in $\sigma$ $[36,37,15]$ :

$$
\mathcal{S}(\sigma)=-g+\frac{c_{\mathrm{s}}}{2} \sigma^{2}+\cdots
$$

The leading constant $g$ is the free energy of stretched torsionally unconstrained DNA of Eq. 2.2. The parameter $c_{\mathrm{s}}$ is the twist stiffness of the extended DNA state and can be measured from the curvature of the extension as a function of $\sigma$ near its maximum. Note that $c_{\mathrm{s}}$ has been defined to have dimensions of energy per length, the same as that of $g$ and force $f$.

The first two terms of the expansion of Eq. 3.7 are sufficient to illustrate the basic properties of state coexistence in a quantitative way. As for the plectonemic state, higher-order terms can be added (e.g. $\sigma^{3}$ to generate positive-negative twisting asymmetry). The most general expansion would replace $\sigma$ with $\sigma-\sigma_{0}$ in Eq. 3.7 where this additional force-dependent $\sigma_{0}$ parameter sets the linking number at which the double helix is relaxed. Variation of $\sigma_{0}$ with force takes into account the stretch-twist coupling. However, for the relatively low forces of interest here (a few $\mathrm{pN}$ ) this coupling has only weak effects and may be neglected (i.e., $\sigma_{0} \approx 0$ ). In any case, $\sigma_{0}$ for a given force is determined by finding the $\sigma$ value at which a local maximum of extension is obtained. Here, $\sigma_{0}=0$ is assumed for the $\mathcal{S}$ state.

Although $c_{\mathrm{s}}$ could be determined directly from experimental data, a theoretical formula does exist based on a large-force perturbative calculation for the semiflexible polymer with harmonic twist rigidity by Moroz and Nelson [36, 37]:

$$
c_{\mathrm{s}}=k_{B} T \omega_{0}^{2} C\left[1-\frac{C}{4 A}\left(\frac{k_{B} T}{A f}\right)^{1 / 2}\right] .
$$

The main effect introduced by this theoretical formula for $c_{\mathrm{s}}$ is a forcedependence of the twisting rigidity. Reducing force reduces $c_{\mathrm{s}}$ since more linking number can be absorbed into writhe fluctuations at lower forces $[36,37]$.

3.3. Free energy of plectonemically supercoiled DNA. A plectonemic supercoil has essentially no extension between its ends, and therefore its free energy has no force dependence (a nonzero force derivative 
would indicate that it has finite extension). In addition, the bare twist energy (2.3) is heavily screened by the large writhing generated by the supercoil $[10,11]$. Given its minimum at $\sigma=0$, the free energy for the plectoneme must have the form

$$
\mathcal{P}(\sigma)=\frac{p}{2} \sigma^{2}+\cdots
$$

The parameter $p$ describes the twist stiffness of the plectonemic state, and like $c_{s}$ and $g$ has dimensions of a force. The stiffness $p$ can be converted to a persistence-like quantity via $p=k_{B} T P \omega_{0}^{2} ; P$ represents the effective twist persistence length of the plectonemic state, accounting for the screening of the bare twist energy (2.3) by the strong writhe of the plectoneme. This screening effect indicates that $P<C$; available data indicate $P \approx 25 \mathrm{~nm}$ [42] for $\approx 100 \mathrm{mM}$ univalent salt in $\mathrm{pH} 7.5$ buffer solution (alternately $\left.p \equiv k_{B} T P \omega_{0}^{2}\right)$.

The stiffness $P$ varies with salt concentration, since that in turn adjusts the effective diameter of the double helix; for higher salt, $P$ is reduced, while for lower salt $P$ increases. This effect arises because the bending energy in a plectonemic superhelix is reduced when the effective diameter is reduced. The torque of the $\mathcal{P}$ state is, by Eq. 3.1, $\tau=\left(p / \omega_{0}\right) \sigma=k_{B} T P \omega_{0} \sigma$.

In Sec. 3.4 only the first, quadratic term of (3.9) is used, which is a reasonable model for $|\sigma|<0.05$, as it allows analytical calculation of all the domain coexistence properties, as well as providing a semi-quantitative description of experiment. However, biochemically it has long been known that anharmonic corrections are present [43]; for large $|\sigma|$ where plectonemic interwinding becomes tight, one can expect appreciable deviations from quadratic behavior. Sec. 4 will show how anharmonicity in (3.9) can modify the extension vs. linking number behavior.

3.4. Analytical results for the harmonic- $\sigma$ state free energies. With the $\mathcal{O}\left(\sigma^{2}\right)$ expressions for plectonemic and extended DNA free energies, the state coexistence behavior at constant force $f$ can be computed following the procedure outlined in Sec. 3.1. The mixed-state free energy is

$$
\mathcal{F}=x_{\mathrm{s}}\left(-g+\frac{c_{\mathrm{s}} \sigma_{\mathrm{s}}^{2}}{2}\right)+x_{\mathrm{p}} \frac{p \sigma_{\mathrm{p}}^{2}}{2} .
$$

Details of the calculation of the equilibrium mixed state can be found in Ref. [27]. This amounts to elimination of $\sigma_{p}$ and $x_{p}$ from (3.10) using $x_{\mathrm{s}}+x_{\mathrm{p}}=1$ and $\sigma=x_{\mathrm{s}} \sigma_{\mathrm{s}}+x_{\mathrm{p}} \sigma_{\mathrm{p}}$, followed by minimization to determine the remaining free parameters $\sigma_{s}$ and $x_{s}$.

The coexisting state linking number densities for this harmonic model work out to be: 


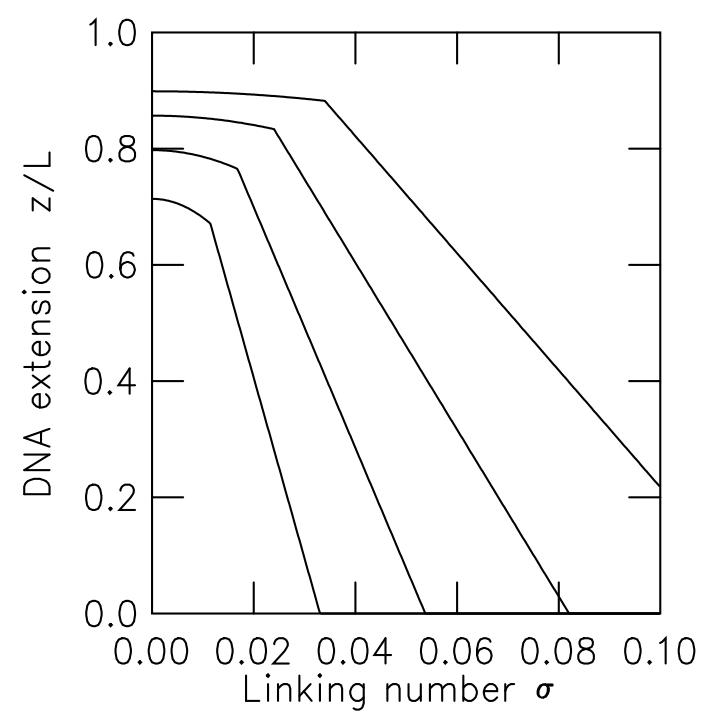

FIG. 3. Extension versus linking number, for forces $0.25 \mathrm{pN}$ (lowest curve), $0.5 \mathrm{pN}$, $1.0 \mathrm{pN}$ and $2.0 \mathrm{pN}$ (highest curve), for positive linking number change $(\sigma>0)$. As force is increased, the extension increases, and the effect of torsional stress (linking number) is reduced. Parameter values are $C=95 \mathrm{~nm}, A=50 \mathrm{~nm}, P=24 \mathrm{~nm}$. The parabolic peak of each extension curve occurs when the DNA is pure extended state; extended and plectonemic DNA are in coexistence on the linear part of each extension curve. The beginning of the linear segments indicates $\sigma_{\mathrm{s}}$, and their $\sigma$-intercepts indicate $\sigma_{\mathrm{p}}$.

$$
\begin{aligned}
& \left|\sigma_{\mathrm{s}}\right|=\frac{1}{c_{\mathrm{s}}}\left(\frac{2 p g}{1-p / c_{\mathrm{s}}}\right)^{1 / 2} \\
& \left|\sigma_{\mathrm{p}}\right|=\frac{1}{p}\left(\frac{2 p g}{1-p / c_{\mathrm{s}}}\right)^{1 / 2} .
\end{aligned}
$$

Since $\sigma_{\mathrm{s}}$ can be determined from experimental data by determining where the linear (coexistence) regime begins, and since the slope $d x_{\mathrm{s}} / d \sigma$ is similarly determined from experimental data, and finally since $g$ is independently well known, it is possible to determine the stiffnesses $c_{\mathrm{s}}$ and $p$. This would amount to a measurement of the free energy of extended and plectonemic DNA in a way relatively independent of details of specific microscopic theories.

Fig. 3 shows extension versus linking number for this model via (3.5) and (3.6). The curves are computed for $f=0.25 \mathrm{pN}$ (lowest curve), $0.5 \mathrm{pN}$, $1.0 \mathrm{pN}$ and $2.0 \mathrm{pN}$ (highest curve). The stiffnesses used were $A=50 \mathrm{~nm}$, $C=95 \mathrm{~nm}$ and $P=24 \mathrm{~nm}$. The parabolic peak at the top of each curve is the region of pure extended DNA. This joins to a linear coexistence 
segment which stretches between $\sigma_{\mathrm{s}}$ and $\sigma_{\mathrm{p}}$, the latter being the point where extension reaches zero. The curves have the characteristic "hat" shape seen experimentally [9].

The equilibrium free energy is easily computed as a function of force and linking number:

$$
\mathcal{F}= \begin{cases}-g+\frac{1}{2} c_{\mathrm{s}} \sigma^{2} & |\sigma|<\left|\sigma_{\mathrm{s}}\right| \\ -g /\left(1-p / c_{\mathrm{s}}\right)+\left[2 p g /\left(1-p / c_{\mathrm{s}}\right)\right]^{1 / 2}|\sigma| & \left|\sigma_{\mathrm{s}}\right|<|\sigma|<\left|\sigma_{\mathrm{p}}\right| \\ \frac{1}{2} p \sigma^{2} & |\sigma|>\left|\sigma_{\mathrm{p}}\right|\end{cases}
$$

In the coexistence region, the linear dependence of the free energy on $\sigma$ indicates that torque $\left(\tau=\omega_{0}^{-1} \partial \mathcal{F} / \partial \sigma\right)$ is a constant. The molecule extension $(X / L=\partial \mathcal{F} / \partial f)$ varies linearly with $\sigma$ in the coexistence region, as anticipated in (3.5).

3.5. DNA torque and its force dependence for the harmonic model. Eq. 3.11 gives the torque, via Eq. 3.1 and either of the pure state free energies Eq. 3.9 or Eq. 3.7:

$$
\tau= \begin{cases}\left(c_{\mathrm{s}} / \omega_{0}\right) \sigma & |\sigma|<\left|\sigma_{\mathrm{s}}\right| \\ \left(2 p g /\left[1-p / c_{\mathrm{s}}\right]\right)^{1 / 2} / \omega_{0} & \left|\sigma_{\mathrm{s}}\right|<|\sigma|<\left|\sigma_{\mathrm{p}}\right| . \\ \left(p / \omega_{0}\right) \sigma & |\sigma|>\left|\sigma_{\mathrm{p}}\right|\end{cases}
$$

For constant force, as $|\sigma|$ is increased from zero, the equilibrium state is first pure extended DNA, with torque growing linearly with $|\sigma|$. Then at $\sigma=\sigma_{\mathrm{s}}$, the coexistence point is reached, and until $\sigma=\sigma_{\mathrm{p}}$ the torque is constant. Finally for $|\sigma|>\left|\sigma_{\mathrm{p}}\right|$, the entire DNA is plectonemic supercoil, and again the torque changes with $|\sigma|$ but at a reduced rate (recall $P<C_{\mathrm{s}}$ ) due to the efficient removal of twist by the large plectonemic writhe.

The middle line of Eq. 3.13 is a main result of this calculation, as it gives the dependence of the coexisting state torque on force:

$$
\tau=\sqrt{\frac{2 k_{B} T P g}{1-P / C_{\mathrm{s}}}} .
$$

This formula is written in terms of the twist persistence length of the plectoneme $(P)$ and of the extended state $C_{\mathrm{s}}=C\left[1-(C / 4 A)\left(k_{B} T / A f\right)^{1 / 2}\right]$ (note $P / C_{\mathrm{s}}=p / c_{\mathrm{s}}$ ).

The force-dependence of the torque enters mainly through the extended state free energy $g=f-\left(k_{B} T f / A\right)^{1 / 2} \approx f$ in the numerator. However, note that the twist persistence length of the extended state $C_{\mathrm{s}}$ increases with increasing force.

The simplest application of Eq. 3.14 is estimating torques in situations where one is in the coexistence range of $\sigma$ and where one knows the force. 
This is commonly the case in magnetic tweezer experiments where force and linking number are both fixed by the position of a macroscopic magnet [9]. An elegant example of use of this is the constant-torque driving of rotational relaxation of DNA [21]. One must be in the coexistence range of $\sigma$ in order to have this constant torque; below $\sigma_{\mathrm{s}}$, the torque will drop below the coexistence value, and above $\sigma_{\mathrm{p}}$ the torque will increase with $|\sigma|$.

It is useful to consider the situation of fixed $\sigma$ and varied force. This is experimentally accessible using various micromanipulation schemes, including magnetic tweezers as long as the force is not reduced to so low a value that linking number can leak away by having the DNA hop over the bead.

Imagine holding $\sigma$ fixed at a value sufficient to form plectonemic supercoils at a low force, and then slowly increase $f$. At low forces, the entire molecule will remain supercoiled (with constant torque $\tau_{\mathrm{p}}=k_{B} T P \omega_{0} \sigma$ until the force reaches a threshold value $f_{\mathrm{p}}$, i.e., until the work done by the external force can overcome the plectoneme's "length binding energy" $[10,11]$. This effect can be analyzed by solving for the force $f_{\mathrm{p}}$ where $x_{\mathrm{s}}=0$, which leads to

$$
g\left(f_{\mathrm{p}}\right)=\frac{p\left[1-p / c_{\mathrm{S}}\right]}{2} \sigma^{2} .
$$

The point $g\left(f_{\mathrm{p}}\right)$ is the minimum extended state free energy (essentially force) needed to start extending the plectonemic DNA. The $f_{\mathrm{p}}$ that solves this equation is the tension inside a plectonemically supercoiled DNA, and for physiological levels of supercoiling $(\sigma=-0.05)$ this force is $\approx 0.5 \mathrm{pN}$.

As force increases further the torque will increase as linking number is shifted increasingly from plectoneme to extended DNA, and the torque will be described by Eq. 3.14. In the coexistence range the torque will be independent of $\sigma$.

Finally, when sufficient force $f_{\mathrm{s}}$ is applied, the plectonemic domains will be destroyed; $f_{\mathrm{s}}$ can be found by solving for when $x_{\mathrm{s}}=1$ :

$$
g\left(f_{\mathrm{s}}\right)=\frac{c_{\mathrm{s}}-p}{2} \sigma^{2} .
$$

The torque at this point will be $\tau_{\mathrm{s}}=\left(c_{\mathrm{s}} / \omega_{0}\right) \sigma$. As force is increased further, the small amount of linking number remaining will be forced into DNA twist, gradually forcing the molecule torque towards its limit of $\tau=$ $k_{B} T C \omega_{0} \sigma$.

Fig. 4 plots torque versus force for $\sigma=0.03,0.04,0.05$ and 0.06 using the same stiffnesses as used in Fig. $3(C=95 \mathrm{~nm}, A=50 \mathrm{~nm}$, $P=24 \mathrm{~nm}$ ). The torque starts at a constant (horizontal segments to left; lowest corresponds to $\sigma=0.03$; highest to $\sigma=0.06)$. Then, the torque starts to increase when extended DNA starts to be created at $f_{\mathrm{p}}$. In the coexistence regime, the torque does not depend on $\sigma$, so all the curves overlap. When $f_{\mathrm{s}}$ is reached, the torque curves separate again, 


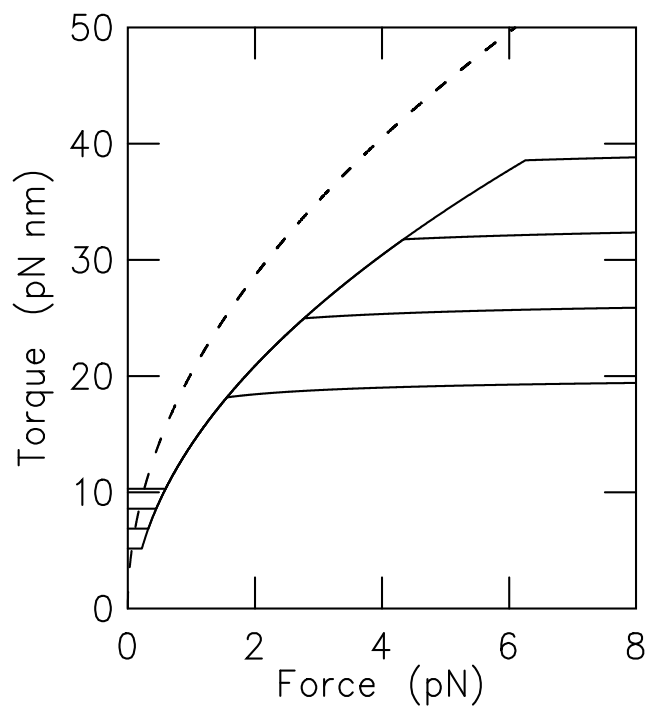

FIG. 4. Torque versus force curve for coexisting state densities corresponding to fixed $\sigma=+0.03,0.04,0.05$ and 0.06, with other parameters as in Fig. 2. For forces below the lower critical forces $f_{\mathrm{p}}$ where the extended DNA state disappears (horizontal line segments at left of graph), torque is constant. Above the upper critical force $f_{\mathrm{s}}$ where the plectonemic DNA disappears, the torque slowly approaches its maximum value $\left(c / \omega_{0}\right) \sigma$ (nearly flat regions to right). Between $f_{\mathrm{p}}$ and $f_{\mathrm{s}}$, the torque follows the same curve for each $\sigma$ value (concave part of curve). Dashed curve shows $\left(2 k_{B} T A f\right)^{1 / 2}$ for comparison.

and then torque only slowly increases with further force increase (nearly horizontal curves to right). These results have been shown to be in good agreement with a numerical calculation of torque in stretched twisted DNA [27] computed using a previously developed Monte Carlo computation [12].

It is worth noting that the torque in the coexistence range of $\sigma(3.14)$ is not equal to $\tau=\left(2 k_{B} T A f\right)^{1 / 2}$ where $A$ is the bending persistence length, as has been suggested in Refs. [8, 9,21]. This formula is reminiscent of that for the critical torque for the linear instability of buckling of a rod under tension $f$ from classical elasticity theory, $\left(4 k_{B} T A f\right)^{1 / 2}[46,36,37,15]$, but with the factor of 4 replaced by a 2 . The derivation of the formula with the 2 uses an approximate calculation [9] based on mechanical energy without including effects of thermal fluctuations.

The formula $\tau=\left(2 k_{B} T A f\right)^{1 / 2}$ (dashed curve in Fig. 4) overestimates the torque calculated above, Eq. 3.14, by roughly $25 \%$. Given the accuracy of single-DNA micromechanical measurements plus the consequences of this overestimate for other measurements e.g., energy landscape parameters for topoisomerases [21], this discrepancy is significant. Experimentally, 


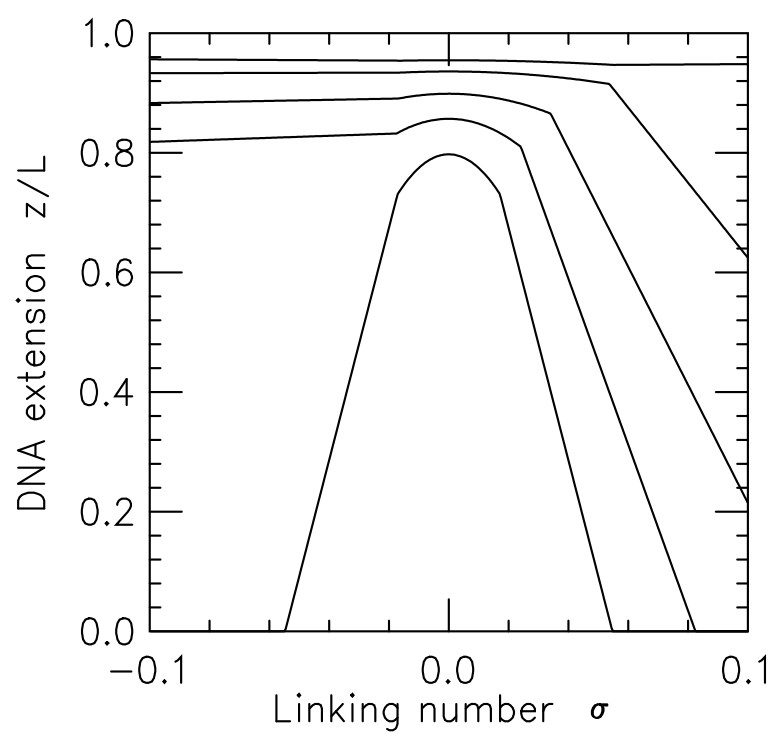

FIG. 5. Extension vs. linking number $\sigma$ for DNA at constant forces, including denaturation effects. Results are shown for forces of $0.5 \mathrm{pN}$ (lowest curve), $1 \mathrm{pN}, 2$ $p N, 5 \mathrm{pN}$ and $10 \mathrm{pN}$ (highest curve), using $A=50 \mathrm{~nm}, C=95 \mathrm{~nm}, P=24 \mathrm{~nm}$, plus description of denaturation as described in Ref. [27]. For $0.5 p N$ the extension is symmetric under sign change of $\sigma$, due to coexistence occurring between extended and plectonemically supercoiled DNA states, which have this symmetry. However, for forces of $1 \mathrm{pN}, 2 \mathrm{pN}$ and $5 \mathrm{pN}$, coexistence occurs between extended and plectonemic DNA for $\sigma>0$, but between extended and denatured DNA for $\sigma<0$, exhibiting a strong breaking of $\sigma \rightarrow-\sigma$ symmetry. Finally for $10 \mathrm{pN}$, denaturation occurs for both positive and negative $\sigma$.

the DNA torque, being dependent on the free energies of the coexisting phases, can be expected to depend on factors which shift around those free energies, notably solution ionic conditions. The torque could be estimated in a model-independent way, using $c_{\mathrm{s}}$ and $g$ extracted directly from experimental data.

3.6. Negative supercoiling and DNA denaturation. The results given in this section are most applicable to positively supercoiled DNA $(\sigma>0)$ where DNA remains in double-helix form for forces up to $\approx 10 \mathrm{pN}$. For $\sigma<0$ the situation is quite different; negative supercoiling of drives denaturation rather readily, leading to ranges with $\sigma<0$ where extension is nearly constant. Denaturation also occurs for positive supercoiling, but only for more extreme values of $\sigma$ [49]. This behavior is straightforward to describe via the framework presented above, by introducing additional pure states corresponding to denatured (strand-separated) DNA [47]. 


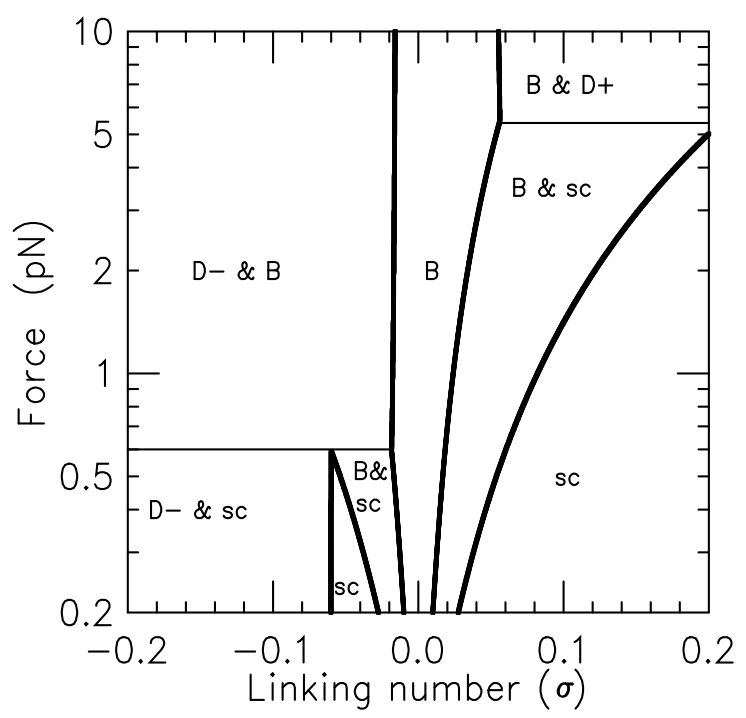

FIG. 6. Force-linking number phase diagram showing transitions between extended double helix (B), plectonemically supercoiled B-DNA (sc), underwound denatured DNA $\left(D_{-}\right)$and overwound denatured DNA $(D+)$. Regions labeled by two pure phases (e.g., " $B$ \& $s c$ ") represent two-phase coexistence regions; thick curves indicate transitions between pure-state and mixed-state regions. The horizontal thin lines are lines of threestate coexistence.

DNA which has been denatured by unwinding has a short persistence length, and has a large negative value of linking number corresponding to the $\sigma \approx-1$ necessary to unwind the double helix. In addition, denatured DNA has a free energy shifted up relative to that of the base-paired DNA double helix, by a sequence-averaged amount of about $2.5 k_{B} T / \mathrm{bp}$ [48]. These effects are discussed in some detail in Ref. [27], including showing comparisons with experimental extension data.

Fig. 5 shows extension versus linking number combining the extended and plectonemic states with denatured states. For $\sigma>0$, coexistence of extended and plectonemically supercoiled DNA occurs as before, but for $\sigma<0$, denatured underwound-melted states preempt the plectonemic state, leading to nearly constant extension with linking number. The nearly constant variation of extension with $\sigma$ once denaturation begins to occur is mainly a consequence of the small amount of the large change of linking number associated with denaturation (complete separation of the DNA strands corresponds $\sigma=-1$ ) combined with the high extensibility of denatured DNA (the DNA backbones can be extended about a factor of two by unwinding them). 
By tabulating the linking numbers at which the various transitions occur, one can construct a force-linking number "phase diagram" (Fig. 6). Since linking number is partitioned between coexisting states, this phase diagram contains "pure state" and "mixed state" regions. In the twostate coexistence regions, torque is constant, and extension varies linearly with $\sigma$. Fig. 6 also contains lines on which three states coexist; the most experimentally accessible of these is the boundary where regions of coexisting extended B-DNA and plectonemic supercoiled DNA ("B \& sc" in Fig. 6) and regions of coexisting B-DNA and denatured underwound DNA ("B \& D-") meet. Notably, there are also regions of denatured overwound DNA, corresponding to the "P-DNA" state observed by Allemand et al. [49]. Ref. [27] presented the corresponding phase diagram plotted in the force-torque plane.

4. Effect of plectonemic free energy anharmonicity on extension of twisted DNA. The harmonic (quadratic- $\sigma$ ) model of Sec. 3.4 has the virtues of being simple and analytically solvable, while still describing experimental data reasonably well [27]. It does suffer from limitations, for example a lack of any accounting for effects of DNA sequence, known to profoundly affect stress-driven DNA denaturation [50]. Also, the basic scheme introduced in Sec. 3.1 whereby two coexisting domains are considered additively could be generalized to include a thermally excited spectrum of "droplets" of the two pure states, along the lines of calculations presented in Ref. [51]. These particular improvements will not be discussed further here.

A limitation of the theory of Sec. 3.4 that will be examined here is the effect of truncation of the pure state free energies at order $\sigma^{2}$. Truncation of the extended state free energy (3.7) at this order does not introduce large errors, since the extended state linking number (for $\sigma>0$ ) is less than $\sigma_{\mathrm{s}}$, the point where plectoneme-extended coexistence begins, and $\sigma_{s}$ remains relatively small. Consistently, experimental data in the pureextended regime $\left(\sigma<\sigma_{\mathrm{s}}\right)$ is well-fit by the extension function resulting from truncation at $\sigma^{2}$, for forces $>0.2 \mathrm{pN}$. For larger forces $(>0.5 \mathrm{pN})$ bending fluctuations in the extended state are suppressed, making the $\sigma^{2}$ term even more dominant over higher-order terms [36] (see Eq. 3.8).

The situation is exactly the opposite for the plectonemic state: the coexistence model indicates that the linking number in the plectonemic domains is never less than $\sigma_{p}$ (for $\sigma>0$ ). Consequently $\sigma_{p}$ is often large: for moderate forces (e.g. $2 \mathrm{pN}) \sigma_{p} \approx 0.1$ (the boundary between "sc \& B" and "sc" in Fig. 6). While the free energy of plectonemic DNA is well described by the initial $\sigma^{2}$ dependence for $\sigma<0.05[43,42,44,45]$, for large $\sigma$ one expects tight interwinding to generate a strong upturn in free energy [11]. It is important to consider the effects of addition of higher-order- $\sigma$ contributions to (3.9). 


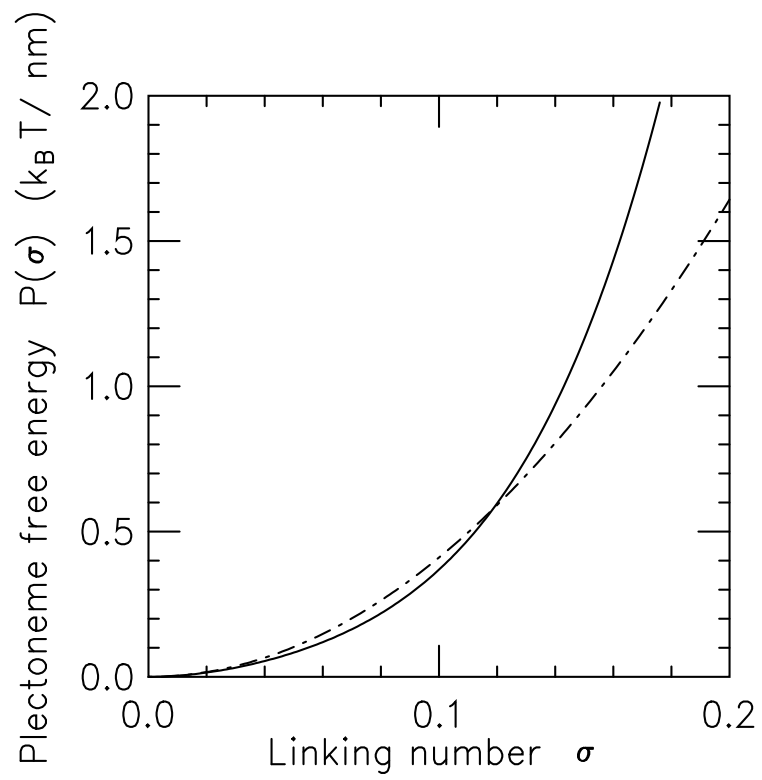

FIG. 7. Harmonic and anharmonic plectonemic free energies. Dashed curve shows purely harmonic plectonemic free energy with $P=24 \mathrm{~nm}$. Solid curve shows anharmonic plectoneme free energy with the same quadratic stiffness $P=24 \mathrm{~nm}$ (recall $\left.p=k_{B} T P \omega_{0}^{2}\right)$ but with nonlinear terms $t=-800 k_{B} T / n m$, and $q=9000 k_{B} T / n m$. The anharmonic free energy has the same initial curvature, but then is softer than the harmonic free energy for intermediate values of $\sigma$. For large $\sigma$ the quartic term makes the anharmonic free energy become greater than the harmonic free energy, providing a model for the breakdown of writhe increase for tight wrapping.

Here anharmonic cubic- and quartic- $\sigma$ terms are added to (3.9):

$$
\mathcal{P}(\sigma)=\frac{p}{2} \sigma^{2}+\frac{t}{3} \sigma^{3}+\frac{q}{4} \sigma^{4} .
$$

This general expansion permits one to have an initial quadratic behavior as required by symmetry, to have a softening of that initial stiffness $(t<0)$ as expected theoretically $[42,11]$, and then to have an upturn in free energy for $\sigma \approx 0.1$ from tight wrapping (Fig. 7).

The free energy model with this addition is

$$
\mathcal{F}=x_{\mathrm{s}}\left(-g+\frac{c_{\mathrm{s}} \sigma_{\mathrm{s}}^{2}}{2}\right)+x_{\mathrm{p}}\left(\frac{p}{2} \sigma_{\mathrm{p}}^{2}+\frac{t}{3} \sigma_{\mathrm{p}}^{3}+\frac{q}{4} \sigma_{\mathrm{p}}^{4}\right) .
$$

This is just (3.10) with cubic and quartic terms added to $\mathcal{P}\left(\sigma_{\mathrm{p}}\right)$. Minimization of this free energy proceeds exactly as in Sec. 3.1 with the change that the calculation cannot be completed in closed form, but can easily be done numerically. 


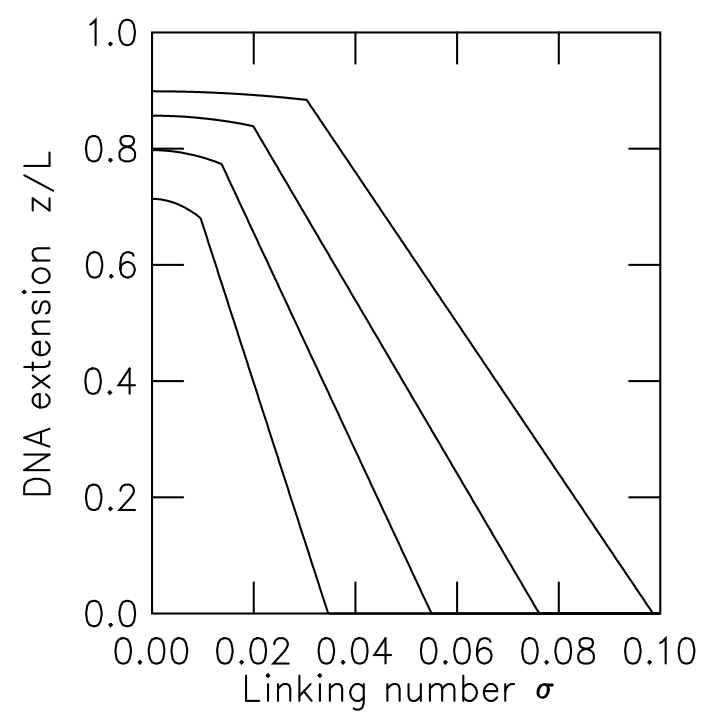

FIG. 8. Extension vs. linking number curves for model of the text, with cubic and quartic term included in the plectoneme free energy. Parameters used are $A=50 \mathrm{~nm}$, $C=95 \mathrm{~nm}, P=24 \mathrm{~nm}, t=-800 k_{B} T / \mathrm{nm}$, and $q=9000 k_{B} T / \mathrm{nm}$ (plectoneme parameters are as in the solid curve of Fig. 7). Curves are plotted for forces of 0.25 (lowest curve), 0.5, 1 and $2 \mathrm{pN}$ (highest curve). These curves should be compared with those of Fig. 3 (see text).

Fig. 8 shows the results of the anharmonic- $\mathcal{P}$ model for extension $v s$. linking number, for the same series of forces $(f=0.25,0.5,1$ and $2 \mathrm{pN})$ and extended-state parameters $(A=50 \mathrm{~nm}, C=95 \mathrm{~nm})$ used in Fig. 3 . The only difference between the calculation leading to Fig. 3 and that leading to Fig. 8 is that for the latter, cubic and quartic terms $(t=-800$ $\left.k_{B} T / \mathrm{nm}, q=9000 k_{B} T / \mathrm{nm}\right)$ corresponding to the solid curve of Fig. 7 have been included. The large numerical values of $t$ and $q$ reflect the fact that the scale for $\sigma$ is $\approx 0.05$.

Comparing Fig. 3 and Fig. 8, the effect of the anharmonic terms in $\mathcal{P}$ is clear. The cubic softening of the plectoneme free energy leads to an increase in the value of $\sigma_{\mathrm{p}}$ for smaller forces, while the quartic stiffening at larger $\sigma$ retards the increase of $\sigma_{\mathrm{p}}$ for larger forces. The result is that the successive extended-plectoneme coexistence curves of Fig. 8 are compressed together, and are more parallel than the rather more splayed ones of Fig. 3. At the same time, the behavior of $\sigma_{s}(f)$ is only mildly affected. Preliminary consideration of available experimental data (see, e.g., left panel of Fig. 3 in Ref. [16]) indicates that this effect will be important to making the theory quantitatively describe experiment. 
Fits of experimental data sets to the theory are still in preparation at the time of this writing, but will be published elsewhere in the near future. It appears likely that this model will be able to arrive at not just values of DNA elastic constants, but will provide insight into the variation of plectonemic supercoil free energy over a wider range of linking number than is accessible using conventional biochemical measurements.

\section{Extrusion of a cruciform from twisted and stretched DNA.} During the workshop, Vincent Croquette discussed experiments whereby special-sequence DNA molecules containing long palindromic repeats were subjected to twisting and pulling [52]. These molecules are able to respond to their underwinding in a way which is not usual for random-sequence DNA, by forming "cruciform" structures, where a region of the palindromic sequence strand-separates, and then where each strand is "extruded" as a hairpin-like double-helical arm. Croquette described how this arrangement may be used to measure the linking number of relaxed DNA, by measuring the length change of the extended region of the DNA per turn introduced, after the cruciform region starts to form. Since a region of DNA must be strand-separated to form the cruciform, this is driven by DNA underwinding $(\sigma<0)$. Croquette and co-workers have shown how this type of molecule may be used to carry out a novel measurement of the helix repeat of relaxed DNA in solution [52].

This situation can also be attacked using the methods presented above, allowing corrections for thermal fluctuations to be calculated. Suppose that a fraction $x_{\mathrm{s}}$ of our molecule is in extended (stretched and twisted) form, while the remaining fraction $x_{\mathrm{c}}=1-x_{\mathrm{s}}$ forms the two hairpin arms. Since the extruded arms are not under any torque or tension, and since they are reconstructed into stretches of double helix, their free energy can be taken to be that of unperturbed double helix, or zero. Thus the free energy of the whole molecule is just that of the extended region:

$$
\mathcal{F}(\sigma)=x_{\mathrm{s}} \mathcal{S}\left(\sigma_{\mathrm{s}}\right)
$$

Since the cruciform arms are entirely unlinked from one another, the linking number of the cruciform region is $\sigma_{\mathrm{c}}=-1$, and since $x_{\mathrm{s}} \sigma_{\mathrm{s}}+x_{\mathrm{c}} \sigma_{\mathrm{c}}=\sigma$, $\sigma_{\mathrm{s}}=\left(1+\sigma-x_{\mathrm{s}}\right) / x_{\mathrm{s}}$. Plugging this into the extended state free energy (3.7) gives

$$
\mathcal{F}(\sigma)=-x_{\mathrm{s}} g+\frac{c_{\mathrm{s}}}{2} \frac{\left(\sigma+1-x_{\mathrm{s}}\right)^{2}}{x_{\mathrm{s}}}
$$

The equilibrium value of $x_{\mathrm{s}}$ is obtained by minimization of (5.2):

$$
x_{\mathrm{s}}=\left\{\begin{array}{cc}
1 & \sigma>\sigma_{c} \\
(1+\sigma) / \sqrt{1-2 g / c_{\mathrm{s}}} & \sigma<\sigma_{c}
\end{array}\right.
$$

where the critical linking number for cruciform extrusion is $\sigma_{\mathrm{c}}=-1+$ $\sqrt{1-2 g / c_{\mathrm{s}}}$. As expected, the critical linking number is negative. 
Recall that $g$ is the free energy per length of untwisted stretched DNA, while $c_{s}$ is the effective twisting stiffness of extended twisted DNA, and that both are essentially known functions of force. For most cases of interest, $g / c_{s}<<1$; for typical forces in the piconewton range, $g \approx k_{B} T / \mathrm{nm}$, while $c_{s}>100 k_{B} T / \mathrm{nm}$. Expansions in $g / c_{s}$ are therefore useful to simplify some of the algebra, as in $\sqrt{1-2 g / c_{\mathrm{s}}} \approx g / c_{\mathrm{s}}$. For example, in this approximation $\sigma_{\mathrm{c}} \approx-g / c_{s}$.

Putting the result (5.3) into (5.2) allows us to calculate the total free energy of the extended-cruciform structure $\left(\sigma<\sigma_{c}\right)$ :

$$
\mathcal{F}(\sigma)=(1+\sigma) c_{\mathrm{s}}\left[\sqrt{1-2 g / c_{\mathrm{s}}}-1\right] \approx-(1+\sigma) g
$$

where the final, approximate term is the leading order in expansion in $g / c_{s}$. The linearity in $\sigma$ indicates that the torque during cruciform formation is constant:

$$
\tau=\frac{1}{\omega_{0}} \frac{\partial \mathcal{F}}{\partial \sigma}=\frac{c_{\mathrm{s}}}{\omega_{0}}\left[\sqrt{1-2 g / c_{\mathrm{s}}}-1\right] \approx-\frac{g}{\omega_{0}} .
$$

Notably, the effect of the twist modulus cancels out of the $\mathcal{O}\left(g / c_{\mathrm{s}}\right)$ result, leaving a dependence of the critical torque for cruciform formation on only the stretching free energy, and on the relaxed B-DNA helix parameter $\omega_{0}$. This could be used to provide a torque standard in the $k_{B} T$ range since both $g$ and $\omega_{0}$ are rather well known $\left(g \approx f \approx k_{B} T / \mathrm{nm}, \omega_{0} \approx 1.85 \mathrm{~nm}^{-1}\right)$. A series of measurements at different forces could therefore be used for a rather precise measurement of $c_{s}(f)$ in the extended state free energy (3.7).

The extension follows as

$$
\frac{X}{L}=-\frac{\partial \mathcal{F}}{\partial f} \approx(1+\sigma) \frac{\partial g}{\partial f}
$$

where only the leading term in expansion in $g / c_{\mathrm{s}}$ is retained for simplicity. Note that the final derivative $\partial g / \partial f$ is just the extension per contour length of untwisted DNA, and therefore that during extended-cruciform coexistence,

$$
\frac{X(f, \sigma)}{X(f, 0)} \approx 1+\sigma=1+\frac{\mathrm{Lk}}{\mathrm{Lk}_{0}}
$$

This leading term of the expansion in $g / c_{\mathrm{s}}$ is essentially Eq. (1) of Ref. [52]. Since the linking number can be counted precisely in terms of magnet turns, the extension change per turn during cruciform formation can therefore be used to measure $\mathrm{Lk}_{0}$, the relaxed linking number of DNA or equivalently the helix repeat of DNA, in solution [52]. The approach presented here provides a thermodynamic derivation which provides corrections due to thermal fluctuations to the analysis in Ref. [52] presented by Vincent Croquette at the workshop. 
One thing that has been omitted in this "intensive" thermodynamic treatment of cruciform formation is the finite free energy cost associated with creating the cruciform; this could be included in the model by adding a constant "core energy" for the cruciform defect to (5.2). Care should be taken with the free energy in this case since it must then be compared to the free energy for pure extended DNA without this core energy present. Extensive pure state free energies should be used since the core energy is a boundary term of $\mathcal{O}\left(k_{B} T\right)$, scaling differently from the total polymer free energy $\mathcal{O}\left(k_{B} T L / \xi\right)$ where $\xi$ is a length comparable to the persistence length. Including this would allow the barrier necessary for cruciform formation to be included in the resulting theory.

6. Conclusion. This paper has reviewed the theory [11, 27] for the elasticity of single twisted DNA molecules, aimed at describing single-DNA micromanipulation experiments. The shape of the extension-linking number curves is characterized by two regimes, a parabolic small- $|\sigma|$ "peak", and for larger $\mid \sigma$, linear "wings". The model discussed in this paper supposes that the peak is "pure" extended DNA, while the wings correspond to "phase coexistence" of an extended state and interwound plectonemic supercoiling $[10,11]$.

While the language of first-order phase transitions is used in this theory, formally there can be no true phase transitions in real experiments on finite-length one-dimensional molecules. However, due to relatively short thermal correlation lengths and appreciable cooperativity, the transitions seen experimentally are remarkably sharp and well described by what amounts to a mean-field theory of first-order transitions.

As discussed in more detail elsewhere [27] this theory gives a good account of experiments where DNA is twisted and pulled [16, 6, 9], and is easily extended to include effects of stress-driven DNA "melting" (strand separation). This paper has reviewed the theory and has presented new results for effects of anharmonicity in the plectoneme free energy, and for the free energy balance during stress-induced formation of cruciform structures in palindromic DNA.

At this point there remain questions concerning the application of this model to experimental data. Careful fits of theory to experiment have not yet been done, and likely will require use of the anharmonic free energy for the plectonemic state. The payoff should be appreciable thanks to the availability of a microscopic model for the extended state of Moroz and Nelson $[36,37]$ which allows measurements of the force-dependent twist rigidity $c_{\mathrm{s}}$ to be linked to the microscopic twist elastic constant $C$.

The coexistence model discussed here allows one to then determine the absolute free energy of plectonemically supercoiled DNA over a very wide range of $\sigma$. The results of Sec. 4 show that even small changes in the shape of the plectonemic free energy $\mathcal{P}(\sigma)$ (Fig. 7) lead to changes in the of the linear portions of the extension vs. linking number curves (compare 
Figs. 3 and 8) that should be readily observable experimentally. Given that salt concentration changes should change the plectonemic free energy more than the extended state free energy, it ought to be possible to use the theory of this paper for stretched twisted DNA to rather comprehensively study the free energy of the interwound plectonemic domains.

The third key output of this theory is prediction of the torque in stretched twisted DNA, and therefore inside plectonemic DNA. Constant torques generated during plectoneme-extended coexistence are starting to be used to drive rotary relaxation experiments on topoisomerases [21, 23], but those torques have proven much more difficult to calibrate than forces. The theory presented here allows one to more directly determine torques in experiments, from extension measurements as a function of force and linking number. Furthermore, molecules containing palindromic sequences suggests may provide a kind of "torque standard", as discussed in Sec. 5.

Many additions, improvements and generalizations of this type of theory are possible. At the workshop Vincent Croquette commented that the behavior of the crude DNA denaturation model [27] used in the model to predict the "phase diagram" and the properties of the denatured states is likely too simple to completely describe experimental data. This issue will require further study, especially of comparison of theory with experimental data, to understand completely. The effects of domain fluctuations and sequence-dependence of denaturation and "pinning" of positions of the plectonemic domains are poorly understood, both experimentally and theoretically. Finally, understanding how the presence of DNA-binding proteins, as found along chromosomes in vivo, modify extension-linking number curves is a subject of theoretical $[53,54]$ and experimental [55] interest.

Acknowlegements. I am indebted to Nick Cozzarelli, Eric Siggia, Vincent Croquette, David Bensimon, Jean-Francois Allemand, Terence Strick, Alfonso Mondragón and Phil Nelson for many collaborations and conversations concerning the topics discussed in this paper, over a period of many years. I am also grateful to Wilma Olson, De Witt Sumners, Craig Benham, and the Institute for Mathematics and its Applications for organizing and sponsoring this workshop in honor of Nick, who was an inspiring leader and colleague. This research was supported by the US National Science Foundation through Grants DMR-9734178, DMR-0203963, MCB-0240998, PHY-0445565, and DMR-0715099, by a subcontract to NIH Grant 1-R21-GM-71019-1, and by awards from Research Corporation, the Petroleum Research Foundation of the American Chemical Society, the Chicago Biomedical Consortium, the University of Illinois Foundation, and the Focused Giving program of the Johnson and Johnson Corporation. 


\section{REFERENCES}

[1] S. Smith, L. Finzi, and C. Bustamante, Science 258, 1122-1126 (1992).

[2] C. Bustamante, J.F. Marko, S. Smith, and E.D. Siggia, Science 265, 1599 1600 (1994).

[3] A.V. VologodskiI, Macromolecules 27, 5623-5625 (1994).

[4] T. OdiJK, Macromolecules 28, 7016-7018 (1995).

[5] J.F. Marko and E.D. Siggia, Macromolecules 28, 8759-8770 (1995).

[6] T.R. Strick, J.-F. Allemand, D. Bensimon, A. Bensimon, and V. Croquette, Science 271, 1835-1837 (1996).

[7] T.R. Strick, J.-F. Allemand, D. Bensimon, and V. Croquette, Biophys. J. 74, 2016-2028 (1998).

[8] T. Strick, J.F. Allemand, D. Bensimon, R. Lavery, and V. Croquette, Physica A 263, 392-404 (1999).

[9] T.R. Strick, J.-F. Allemand, V. Croquette, and D. Bensimon, Prog. Biophys. Mol. Biol. 74, 115-140 (2000).

[10] J.F. Marko And E.D. Siggia, Science 265, 506-508 (1994).

[11] J.F. Marko and E.D. Siggia, Phys. Rev. E 52, 2912-2938 (1995).

[12] J.F. Marko and A. VologodskiI, Biophys. J. 73 123-132 (1997).

[13] B. Fain, J. Rudnick, and S. Ostlund, Phys. Rev. E 55, 7364-7368 (1997).

[14] J.F. Marko, Phys. Rev. E 55, 1758-1772 (1997).

[15] J.F. Marko, Phys. Rev. E 57, 2134-2149 (1998).

[16] S. Neukirch, Phys. Rev. Lett. 93, 198107 (2004).

[17] T.R. Strick, V. Croquette, And D. Bensimon, Nature 404, 901-904 (2000).

[18] N.J. Crisona, T.R. Strick, D. Bensimon, V. Croquette, and N.R. Cozzarelli, Genes. Dev. 14, 2881-2892 (2000).

[19] N.H. Dekker, V.V. Rybenkov, M. Duguet, N.J. Crisona, N.R. Cozzarelli, D. Bensimon, and V. Croquette, Proc. Natl. Acad. Sci. USA 99 12126-12131 (2002).

[20] G. Charvin, T.R. Strick, D. Bensimon, and V. Croquette, Biophys. J. 89 384-392 (2005).

[21] D.A. Koster, V. Croquette, C. Dekker, S. Shuman, and N.H. Dekker, Nature 434 671-674 (2005).

[22] N.H. Dekker, T. Viard, C.B. De la Tour, M. Duguet, D. Bensimon, and V. Croquette, J. Mol. Biol. 329 271-282 (2003).

[23] B. Taneja, B. Schnurr, A. Slesarev, J.F. Marko, and A. Mondragon, Proc. Natl. Acad. Sci. USA 104, 14670-14675 (2007).

[24] Z. Bryant, M.D. Stone, J. Gore, S.B. Smith, N.R. Cozzarelli, and C. Bustamante, Nature 424, 338-341 (2003).

[25] J. Gore, Z. Bryant, M.D. Stone, M.N. Nollmann, N.R. Cozzarelli, and C. Bustamante, Nature 439 100-104 (2006).

[26] C. Deufel, S. Forth, C.R. Simmons, S. Dejgosha, and M.D. Wang, Nature Methods 4 223-225 (2007).

[27] J.F. Marko, Phys. Rev. E 76, 021926 (2007).

[28] J.F. MARKo, in Les Houches Session LXXXII, Multiple aspects of DNA and RNA from biophysics to bioinformatics, ed. D. Chatenay et al., pp. 248-250 (Elsevier, San Diego CA, 2005).

[29] P.J. Hagerman, Ann. Rev. Biophys. Biophys. Chem. 17 265-86 (1988).

[30] P. Cluzel, A. Lebrun, C. Heller, R. Lavery, J.L. Viovy, D. Chatenay, and F. Caron, Science 271, 792-794 (1996).

[31] S.B. Smith, Y. Cui, and C. Bustamante, Science 271, 795-9 (1996).

[32] R.W. Ogden, G. Saccomandi, and I. Sgura, Comp. Math. Appl. 53, 276-286 (2007).

[33] L.D. Landau and E.M. Lifshitz, Theory of Elasticity, Ch. II (Pergamon, New York NY, 1986). 
[34] D.M. Crothers, J. Drak, J.D. Kahn, and S.D. Levene, Meth. Enzym. 212 3-29 (1992).

[35] V. Rossetto, Europhys. Lett. 69 142-148 (2005).

[36] J.D. Moroz and P. Nelson, Proc. Natl. Acad. Sci. USA 94, 14418-14422 (1997)

[37] J.D. Moroz And P. Nelson, Macromolecules 31, 6333-6347 (1998).

[38] J.F. MARKO, Europhys. Lett. 38, 183-188 (1997).

[39] R.D. Kamien, T.C. Lubensky, P. Nelson, and C.S. O'Hern, Europhys. Lett. 38, 237-242 (1997).

[40] T. Lionnet, S. Joubaud, R. Lavery, D. Bensimon, and V. Croquette, Phys. Rev. Lett. 96, 178102 (2006).

[41] J. Gore, Z. Bryant, M. Nollmann, M.U. Le, N.R. Cozzarelli, and C. BusTAMANTE, Nature 442, 836-839 (2006).

[42] A.V. Vologodskit, S.D. Levene, K.V. Klenin, M. Frank-KamenetskiI, and N.R. Cozzarelli, J. Mol. Biol. 227, 1224-1243 (1992).

[43] W. Bauer and J. Vinograd, J. Mol. Biol. 47, 419-35 (1970).

[44] K.V. Klenin, A.V. Vologodskit, V.V. Anshelevich, A.M. Dykhne, and M.D. Frank-Kamenetskit, J. Mol. Biol. 217, 413-419 (1991)

[45] V.V. Rybenkov, A.V. Vologodkskit, and N.R. Cozzarelli, Nucl. Acids Res. 25 1412-1418 (1997).

[46] A.E.H. Love, A Treatise on the mathematical theory of elasticity, pp. 417-419 (Dover, New York NY, 1944)

[47] A. Sarkar, J.F. Léger, D. Chatenay, and J.F. Marko, Phys. Rev. E 63051903 (2001).

[48] S. Cocco, J. Yan, J.F. Léger, D. Chatenay, and J.F. Marko, Phys. Rev. E 70011910 (2004).

[49] J.F. Allemand, D. Bensimon, and V. Croquette, Proc. Natl. Acad. Sci. USA 95, 14152-14157 (1998).

[50] T.R. Strick, V. Croquette, and D. Bensimon, Proc. Natl. Acad. Sci. USA 95 10579-10583 (1998)

[51] S. Kutter and E.M. Terentuev, Eur. J. Phys. B 21, 455-462 (2001); S. Kutter, Ph.D. Thesis, (University of Cambridge, England UK, 2002).

[52] A. Dawid, F. Guillemot, C. Breème, V. Croquette, and F. Heslot, Phys. Rev. Lett. 96, 188102 (2006).

[53] J. Yan and J.F. Marko, Phys. Rev. E 68, 011905 (2003).

[54] S. Cocco, J.F. Marko, R. Monasson, A. Sarkar, and J. Yan, Eur. Phys. J. E 10, 249-263 (2003).

[55] B. Schnurr, C. Vorgias, and J. Stavans, Biophys. Rev. Lett. 1, 29-44 (2006). 